\title{
PULSE-ECHO FIELD DISTRIBUTION MEASUREMENT TECHNIQUE FOR HIGH-FREQUENCY ULTRASOUND SOURCES
}

\author{
Kay Raum and William D. O'Brien, Jr.
}

Bioacoustics Research Laboratory, Beckman Institute for Advanced Science and Technology, Department of Electrical and Computer Engineering, University of Illinois, 405 North Mathews Avenue, Urbana, IL 61801 (Current address for KR: Institute of Medical Physics and Biophysics, Martin-Luther-University Halle-Wittenberg, D-06097 Halle/Saale, Germany)

Abstract - A simple technique for the determination of the spatial and temporal transmit-receive field distribution of spherically focused highfrequency transducers is described. In this study tungsten wires were used as echo-targets. Based on the scattering of sound on a rigid cylinder the transmit-receive field projection of spherical sources measured with a wire target was compared with both, theoretical pressure distributions and hydrophone measurements in the frequency range from 3 to $17 \mathrm{MHz}$. It was demonstrated that both techniques yielded comparable results for the low-frequency transducer, whereas only the results of the wire target technique were also in agreement with theory for the higher frequency transducers.

\section{INTRODUCTION}

The use of spherically-focused, high-frequency transducers is widespread in many high-resolution ultrasonic imaging applications such as in medical diagnosis and material investigations.

In this contribution a simple transmit-receive field projection technique is proposed to estimate spatial and temporal field quantities of focused high-frequency transducers. Tungsten wires with diameters less than the acoustic wavelength were used as echo-targets. Using a small-diameter wire instead of a point-like target enables one to choose a target size (wire diameter) smaller than the acoustic wavelength, even for higher frequencies. This provides good spatial (in axial and lateral scan direction) and temporal resolution, while the received signal amplitude is still sufficient due to the larger target area which is oriented perpendicular to the lateral scan direction and the beam axis, respectively. Additionally, as long as an axial-symmetric field distribution can be assumed only two scan directions are necessary for a spatial field projection.
As long as the wire is sufficient small $\left(k a^{\prime}<1\right.$, where $k$ is the wave number and $a^{\prime}$ is the radius of the wire) resonance modes are not excited by an incident wave and the wave scattered by the cylinder has the form [12]

$$
\begin{array}{r}
p_{s} \rightarrow \sqrt{\frac{2}{\pi i k r} \exp \left[j\left(k_{z} z+k_{i} r\right)\right] \frac{i \pi k_{i}^{2} a^{\prime 2}}{4}} \\
\cdot\left(\frac{\kappa_{e}-\kappa}{\kappa}+\frac{2\left(\rho_{e}-\rho\right)}{\rho} \cos \phi\right)
\end{array}
$$

where $\kappa$ are the compressibilities and $\rho$ are the densities, respectively.

The lateral acoustic pressure distribution in the focal plane of a spherical focusing source can be described by $[10,11]$

$$
\left|\frac{p(r)}{p(0)}\right|=\left|\frac{2 J_{1}\left(\frac{k a r}{R O C}\right)}{\frac{k a r}{R O C}}\right|,
$$

where $p(r)$ is the peak acoustic pressure as a function of the off-axis lateral distance $r, p(0)$ is the on-axis peak acoustic pressure at $z=R O C, k$ is the wave number, $a$ is the radius of the transducer and $R O C$ is the transducer's radius of curvature. $J_{l}(x)$ is the Bessel function of the first kind of order one.

The axial pressure distribution of a spherical focusing source is described by $[10,11]$

$$
\left|\frac{p(z)}{p(0)}\right|=\frac{R O C}{z}\left|\operatorname{Sind}\left(\frac{a^{2}}{2 \lambda R O C}\left(\frac{R O C}{z}-1\right)\right)\right| \text {, }
$$

where $p(z)$ is the peak acoustic pressure as a function of the axial distance $z$. 
For a pulse-echo wire target, the line integral of the point-to-point distribution is obtained, that is the pressure projection $f_{p}(x, z)$ :

$$
f_{p}(x, z)=\int p(x, y, z) d y
$$

where the wire is parallel to the $y$ axis. The geometry dictates that the beam must be symmetric about the beam axis and have relatively weak side lobes in order to properly characterize the field's dimensions near the beam axis. These conditions are satisfied for strongly focused beams from a spherical transducer. Since the pulse-echo field is composed of the multiplication of the transmitted and received fields, equations (2) to (4) have to be squared for the distributions of the transmitreceive field.

\section{EXPERIMENTS}

Tungsten wire targets with different diameters $(25,37,63,80 \mu \mathrm{m}$; California Fine Wire Company, Grover City, CA) were placed in a tank filled with distilled, degassed water $\left(\approx 20^{\circ} \mathrm{C}\right)$ and oriented normal to the sound beam direction (Figure 1). The targets were scanned across the acoustic field using a computer-controlled microprecision positioning system (Daedal Inc., Harrison City, PA) with positional accuracy of about $2 \mu \mathrm{m}$. The grid size spacings in the lateral and axial directions were $25 \mu \mathrm{m}$ and $50 \mu \mathrm{m}$. The transducers were excited by a $300 \mathrm{~V}$ mono-cycle pulse produced by a computer-controlled pulser/receiver (Model 5800; Panametrics, Waltham, MA). The received signal was amplified $(20 \mathrm{~dB})$ and band-pass filtered (1-35 MHz) by the pulser/receiver. For all measurements, the signal was displayed $(500 \mathrm{Ms} / \mathrm{s})$ on a digitizing oscilloscope (Model 11401; Tektronix) with a 10bit resolution. Positioning system, oscilloscope

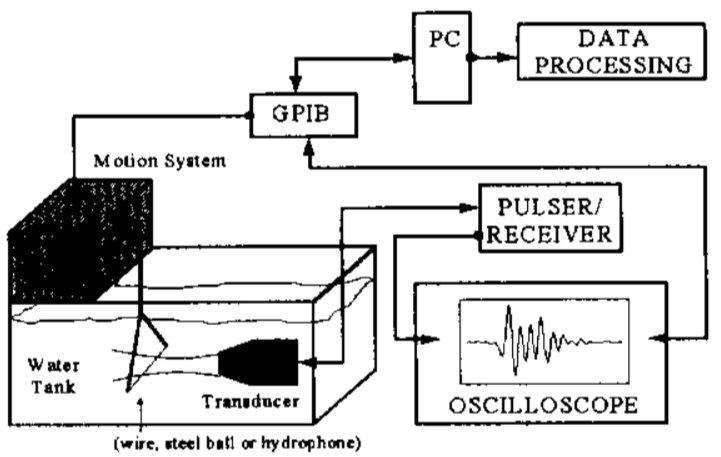

Fig. 1. Block diagram of the main system components and pulser/receiver were connected to a GPIBboard and controlled by a 486-66 PC. The time window at the oscilloscope was moved with every axial scan movement to maintain a high sampling rate $(500 \mathrm{Ms} / \mathrm{s})$ with a low number of sample points. Each 512-point RF-signal was stored to the hard disk and transferred to a SUN Sparc 20 workstation for off-line processing. For comparison the transmitted field distribution was measured with a calibrated PVDF bilaminar membrane hydrophone with an effective diameter of $0.785 \pm 0.007 \mathrm{~mm}$ (Model 804, Sonic Industries, Hatboro, PA). All computations were performed with MATLAB ${ }^{\circledR}$ (The MathWorks, Inc., Natick, MA).

2D - field projections were obtained from the high frequency probes using a $25 \mu \mathrm{m}$ tungsten wire (Figure 2). In the focal plane the lateral field distribution was also assessed from all transducers using different wire diameters and a hydrophone, respectively. All spatial graphs are plotted as the transmit-receive distribution of the pulse intensity integral $(P I I)$ in $\mathrm{dB}$. The frequency spectra obtained with a $25 \mu \mathrm{m}$ wire at the focal point were used to estimate the theoretical lateral/axial distributions with eq. (2), (3) and (4). In order to simulate a transmit-receive field the calculated as well as the hydrophone determined distributions were squared (Fig. 3).

Pulse duration $\left(\tau_{(-20 d B)}\right)$, bandwidth $(\Delta f)$, center frequency $\left(f_{c}\right)$ and fractional bandwidth were determined from the RF-signal at the focal point.

\section{RESULTS}

Figure 2 shows a transmit - receive field projection of the $20 \mathrm{MHz}$-probe obtained with a 25 $\mu \mathrm{m}$ wire. Each contour line represents a $3 \mathrm{~dB}$ decrease of the $P I I$ value. The true focal length was determined from the on-axis time-of-flight of the received signal at the maximum $P I I$ location. The measured focal length $(F)$, depth of focus $\left(F_{z}\right)$ and beam width $\left(D_{\text {lateral }}\right)$ at the focal plane are comparable to the calculated values (Figure 2 and Table I).

Pulse duration, bandwidth and center frequency were obtained from the true focal point RF signal using a $25-\mu \mathrm{m}$ wire for the high frequency probes and a $80-\mu \mathrm{m}$ wire for the $3 \mathrm{MHz}$ probe, respectively (Figure 3 ). It was found that the 35 $\mathrm{MHz}$ pulser/receiver used in this study appeared to attenuate the higher frequency components 


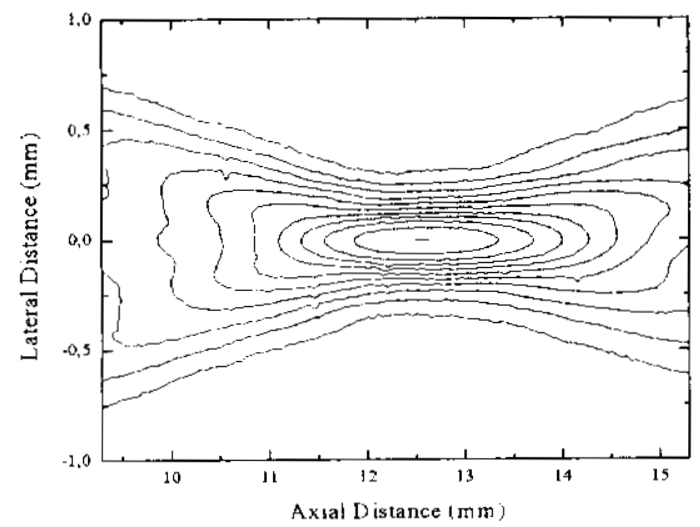

Fig. 2. Contour plot of the spatial intensity distribution $(P I I$ in $\mathrm{dB}$ ) of the $20 \mathrm{MHz}$ transducer field. Each contour line indicates a decrease of the PII of $3 \mathrm{~dB}$

which resulted in the estimated center frequencies of the two higher frequency probes being considerably lower than the manufacturer-stated frequencies, that is, $13.05 \mathrm{MHz}$ for the $15-\mathrm{MHz}$ transducer and $17.33 \mathrm{MHz}$ for the $20-\mathrm{MHz}$ transducer. A comparison of wire determined lateral/axial field distributions (wire (M)) with theoretical results (point (TH) and wire (TH)) shows very good agreement near the focal region $(P I I>-10 \mathrm{~dB})$. However, the squared hydrophone determined lateral distribution (hydrophone $(M)$ ) in the focal plane is in agreement with theoretical/wire determined results for the $3 \mathrm{MHz}$ probe only. This demonstrates that the hydrophone's effective diameter is dominant over that of the field's actual lateral distribution when the target size is larger than significant spatial changes of the field's pressure distribution.

Different wire diameters did not have an appreciable effect on the measured beam width (mean agreements to within $6.8,6.6$ and $2.9 \%$ for the 3-, $15-$ and $20 \mathrm{MHz}$ transducers, respectivcly). All measured lateral beam distributions were in the range between the calculated wire projections and the point-to-point distributions (near the beam axis), and therefore, the experimental observations support the suggestion that a wire diameter less than the acoustic wavelength is sufficient to obtain the significant field dimensions for spherically focused fields.

\section{CONCLUUSION}

The results demonstrate that the wire-target technique is a simple and powerful measurement procedure to determine the spatial and temporal transmit-receive acoustic field quantities from high-frequency sources when an appropriately small effective hydrophone diameter is not available. For the low-frequency case investigated herein, the hydrophone and wire-target techniques yielded comparable results. For the two highfrequency cases, the wire-target

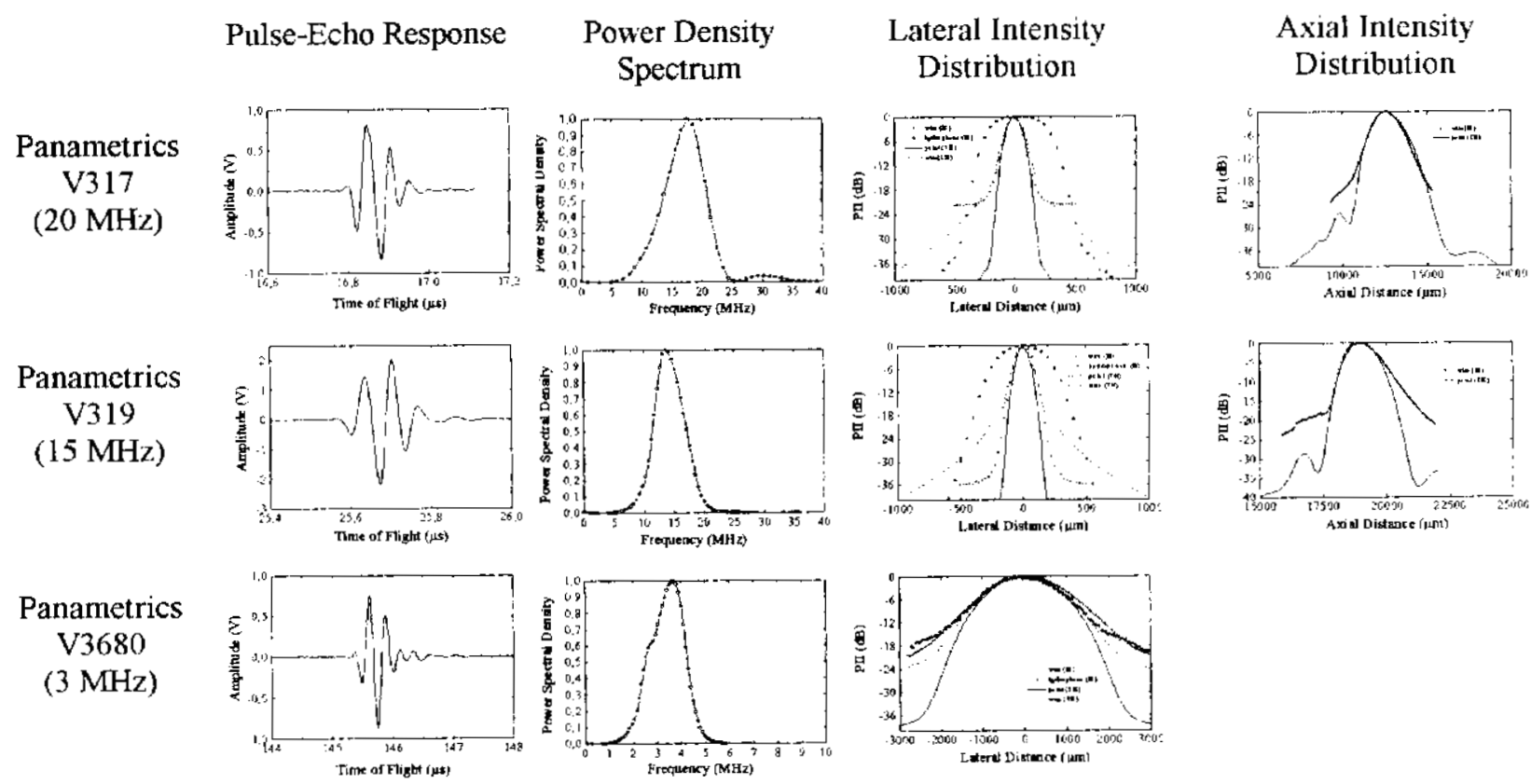

Fig. 3. Pulse-echo response, power density spectrum, lateral and axial intensity distributions. All graphs are plotted as transmit-receive distributions. 
TABLE I MEASURED SPATIAL AND TEMPORAL FIELD QUANTITIES USING A $25-\mu \mathrm{m}$ WIRE TARGET. WhERE APPLICABLE, COMPARISON OF MEASURED QUANTITIES WITH MANUFACTURER'S CERTIFICATION OR CALCULATED VALUES IS PROVIDED. (CALCULATED VALUES ARE DENOTED WITH AN * AND A BLANK INDICATES EITHER THE INFORMATION WAS NOT MEASURED OR THE MANUFACTURER DID NOT PROVIDE THE INFORMATION.)

\begin{tabular}{lcccccc}
\hline \multirow{2}{*}{$\begin{array}{c}\text { Transducer } \\
\text { or field quantity }\end{array}$} & \multicolumn{2}{c}{ V3680 3-MHz Transducer } & V319 15-MHz Transducer & V317 20-MHz Transducer \\
\cline { 2 - 6 } & Measured & $\begin{array}{c}\text { Certification/ } \\
\text { Calculated* }\end{array}$ & Measured & $\begin{array}{c}\text { Certification/ } \\
\text { Calculated* }\end{array}$ & $\begin{array}{c}\text { Measured } \\
\text { Certification/ } \\
\text { Calculated* }\end{array}$ \\
\hline$F$ & & $100 \mathrm{~mm}$ & $18.70 \mathrm{~mm}$ & $19.05 \mathrm{~mm}$ & $12.44 \mathrm{~mm}$ & $12.70 \mathrm{~mm}$ \\
$f_{\text {-number }}$ & & $10 \mathrm{~mm}$ & & $6.35 \mathrm{~mm}$ & & $3.175 \mathrm{~mm}$ \\
$F_{\mathrm{Z}}$ & & 5 & 1.5 & 2 \\
$D_{\text {lateral }}$ & & $72.9 \mathrm{~mm}^{*}$ & $1.80 \mathrm{~mm}$ & $1.81 \mathrm{~mm}^{*}$ & $2.15 \mathrm{~mm}$ & $2.42 \mathrm{~mm}^{*}$ \\
$f_{1}$ & $2.3 \mathrm{~mm}$ & $2.1 \mathrm{~mm}^{*}$ & $187 \mu \mathrm{m}$ & $175 \mu \mathrm{m}^{*}$ & $173 \mu \mathrm{m}$ & $176 \mu \mathrm{m}^{*}$ \\
$f_{2}$ & $2.70 \mathrm{MHz}$ & & $11.05 \mathrm{MHz}$ & $10.60 \mathrm{MHz}$ & $13.65 \mathrm{MHz}$ & $15.80 \mathrm{MHz}$ \\
$f_{C}$ & $4.45 \mathrm{MHz}$ & & $15.05 \mathrm{MHz}$ & $19.80 \mathrm{MHz}$ & $21.00 \mathrm{MHz}$ & $27.70 \mathrm{MHz}$ \\
$\Delta f$ & $3.60 \mathrm{MHz}$ & & $13.05 \mathrm{MHz}$ & $15.20 \mathrm{MHz}$ & $17.33 \mathrm{MHz}$ & $21.75 \mathrm{MHz}$ \\
Fractional Bandwidth & $1.75 \mathrm{MHz}$ & & $4.00 \mathrm{MHz}$ & $9.20 \mathrm{MHz}$ & $7.35 \mathrm{MHz}$ & $5.95 \mathrm{MHz}$ \\
$t_{(-20 \mathrm{~dB})}$ & $48.6 \%$ & & $30.7 \%$ & $60.5 \%$ & $42.4 \%$ & $27.4 \%$ \\
\hline
\end{tabular}

technique yielded spatial field information more consistent with calculated quantities. Also, from the wire-target reflected waveform from the focal point, appropriate temporal field quantities can be determined. In principal there are no upper frequency limits for the wire-target technique as long as the wire diameter is smaller than the acoustic wavelength and the signal-to-noise is adequate.

[This work was supported in part by the OttoRitter-Stiftung.]

\section{REFERENCES}

[1] K. Raum and W. D. O'Brien, Jr., "Pulse-echo field distribution measurement technique for high-frequency ultrasound sources," IEEE Trans. Ultrason., Ferroelect., Freq. Contr., vol. 44, pp. 810-815, July 1997.

[2] G. E. Tupholme, "Generation of acoustic pulses by baffled plane pistons," Mathematika, vol. 16, pp. 209-224, 1969.

[3] P. R. Stephanishen, "The time-dependent force and radiation impedance on a piston in a rigid infinite planar baffle," J. Acoust. Soc. Amer., vol. 49, no. 3, pp. 841-849, 1971.

[4] P. R. Stephanishen, "Transient radiation from pistons in a infinite planar baffle," J. Acoust. Soc. Amer., vol. 49, pp., no. 5, pp. 1629-1638, 1971.

[5] B. Schneider and K. K. Shung, "Quantitative analysis of pulsed ultrasonic beam patterns using a Schlieren system," IEEE Trans. Ultra- son. Ferroelect. Freq. Contr., vol. 43, no. 6, pp 1181-1186, 1996.

[6] D. R. Bacon, "Characteristics of a PVDF membrane hydrophone for use in the range 1$100 \mathrm{MHz}$, " IEEE Trans. on Sonics and Ultrasonics, vol. SU-29, pp. 18-25, 1982.

[7] C. A. Bernier, "A practical approach to measuring an intravascular ultrasonographic imaging system beam pattern," J. Ultrasound Med., vol. 14, pp. 367-372, 1995.

[8] T. Li and M. Ueda, "Sound scattering of a plane wave obliquely incident on a cylinder," J. Acoust. Soc. Amer., vol. 86, no. 6, pp. 2363-2368, 1989.

[9] T. Li, H. Shimamoto and M. Ueda, "Measurement of transmit-receive sound intensity pattern of ultrasound transducer using echoes scattered by a fine wire," J. Acoust. Soc. Jpn., vol. 46, no. 10, pp. $810816,1990$.

[10] Lucas and Muir, "The field of a focusing source," J. Acoust. Soc. Am., vol. 72, pp. 1289-96, 1982.

[11] G. S. Kino, Acoustic Waves: Devices, Imaging, and Analog Signal Processing. Englewood Cliffs, NJ: Prentice-Hall, Inc., 1987.

[12] Ph. M. Morse and K. U. Ingard, Theoretical Acoustics. New York, McGraw-Hill, Inc., 1968.

[13] Acoustic output measurement and labeling standard for diagnostic ultrasound equipment. Laurel, MD: American Institute of Ultrasound in Medicine, 1992.

[14] W. L. Nyborg, Intermediate Biophysical Mechanics. Menlo Park, CA: Cummings Publishing Co., 1975. 